\title{
Successful Management with CHOP for Pulmonary Lymphomatoid Granulomatosis in a Dog
}

\author{
Singo HATOYA ${ }^{1)}$, Daijiro KUMAGAI ${ }^{1)}$, Seiko TAKEDA ${ }^{1)}$, Emi YAMAMOTO ${ }^{2}$, Masako NAKANISHI ${ }^{2}$, \\ Mitsuru KUWAMURA ${ }^{2) *}$, Kikuya SUGIURA ${ }^{1)}$, Hiroshi SASAI ${ }^{3)}$, Jyoji YAMATE $^{2)}$ and Toshio INABA ${ }^{1)}$ \\ Departments of ${ }^{1)}$ Advanced Pathobiology and ${ }^{2}$ Integrated Structural Biosciences, Graduate School of Life and Environmental Sciences, \\ Osaka Prefecture University, 1-58 Rinku Ourai Kita, Izumisano, Osaka 598-8531 and ${ }^{3)}$ Kitasuma Animal Hospital, 9-5-8 Yokoo, \\ Sumaku, Kobe, Hyogo 654-0131, Japan
}

(Received 12 July 2010/Accepted 22 November 2010/Published online in J-STAGE 6 December 2010)

ABSTRACT. A 3-year-old, spayed female miniature dachshund was presented for vomiting and anorexia. Thoracic radiographs and CT scan revealed abnormal pulmonary opacities at bilateral caudal lobe. Cytological analysis of the pulmonary mass revealed the presence of large lymphohistiocytic cells and small lymphocytes with occasional neutrophils and plasma cells. An open lung biopsy was performed and a diagnosis of pulmonary lymphomatoid granulomatosis (LYG) was made. The dog was administered CHOP based therapy (modified UW-25), and it survived for 1,022 days after admission. Immunohistochemistry revealed pulmonary lesions consisted of many CD79a positive B cells aggregation and proliferation with prominent angiocentric pattern. This was the first case of canine pulmonary LYG managed by CHOP chemotherapy.

KEY WORDS: canine, CHOP, lymphomatoid granulomatosis, pulmonary.

Lymphomatoid granulomatosis (LYG) is a unique form of pulmonary angiitis and granulomatosis which was first described by Liebow et al. in 1972 [12]. Histologically it is a necrotizing angiocentric and angiodestructive infiltrative process composed of small lymphocytes, plasma cells, histiocytes and atypical lympho-reticular cells $[11,12]$. In veterinary medicine, canine pulmonary LYG is a rare pulmonary lymphoproliferative disease; very few treatment studies have been conducted and no standard treatment is established [2, 17]. In this report, we describe clinical and pathological findings of a canine LYG, which results in long-term survive with CHOP therapy, modified version of the University of Wisconsin-Madison (UW-25) protocol for canine lymphoma [7].

A 3-year-old, spayed female miniature dachshund, weighing $3.5 \mathrm{~kg}$, was presented to Osaka Prefecture University Veterinary Clinical Center for 4 week-history of vomiting and anorexia. Clinical examination revealed pyrexia and dyspnea. A peripheral lymphadenopathy was not recognized. There was no evidence of hepatomegaly or splenomegaly. A complete blood count showed abnormal leukocytosis $(40,100 \mathrm{cell} / \mu l$; reference range 6,000 to $17,000 / \mu l)$ with mature neutrophilia $(23,258 \mathrm{cell} / \mu l$; reference range 3,000 to $11,500 / \mu l)$ and lymphocytosis $(16,441$ cell $/ \mu l$ reference range 1,000 to $4,800 / \mu l$ ). The blood biochemical findings included an increased total protein concentration $(8.4 \mathrm{~g} / \mathrm{d} l)$ with a high globulin concentration (A/ $\mathrm{G}=0.5)$. Increased $\alpha-2$ and $\gamma$ fraction were identified via serum electrophoresis. The dog was negative for Dirofi-

\footnotetext{
* Correspondence to: Kuwamura, M., Department of Integrated Structural Biosciences, Graduate School of Life and Environmental Sciences, Osaka Prefecture University, 1-58 Rinku Ourai Kita, Izumisano, Osaka 598-8531, Japan.

e-mail: kuwamura@vet.osakafu-u.ac.jp
}

laria immitis infestation. Thoracic radiographs revealed abnormal pulmonary opacities at bilateral caudal lobe (Fig. 1). Radiographic evidence of sterna lymphadenopathy was also noted. A chest computed tomographic (CT) scan of the thorax showed bilateral, wedge-shaped opacities (Fig. 2). Cytological analysis of the pulmonary mass, which was performed by fine-needle aspiration revealed the presence of large lymphohistiocytic cells and small lymphocytes with occasional neutrophils and plasma cells. The dog was treated with Enrofloxacin (5 mg/kg po SID) and Cephalexin (25 $\mathrm{mg} / \mathrm{kg}$ po BID) from first admission. Although the number of neutrophil and lymphocyte was normalized, clinical signs did not resolved by antibiotics therapy. In an attempt to obtain a diagnosis, an open lung biopsy was performed in the left caudal lobe field on day 20 after admis-

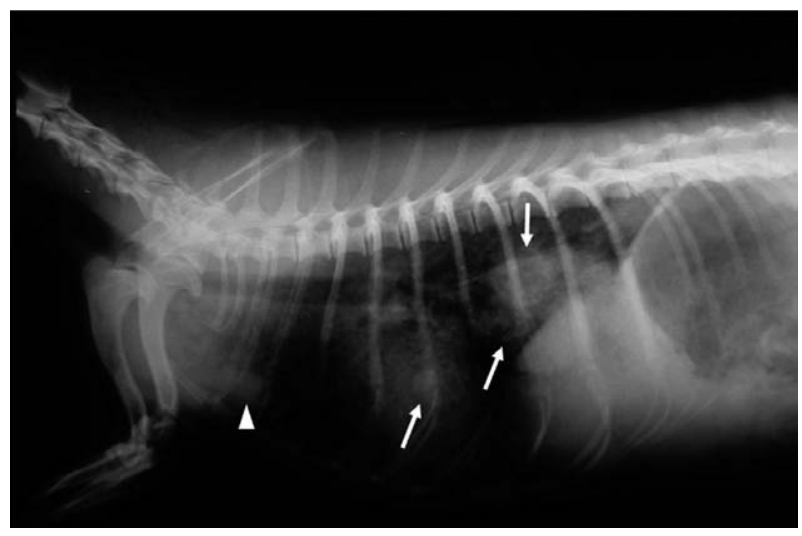

Fig. 1. Thoracic radiograph showing the presence of sterna lymphadenopathy (arrowhead) and abnormal pulmonary opacities (arrows) at bilateral caudal lobe (left lateral) 


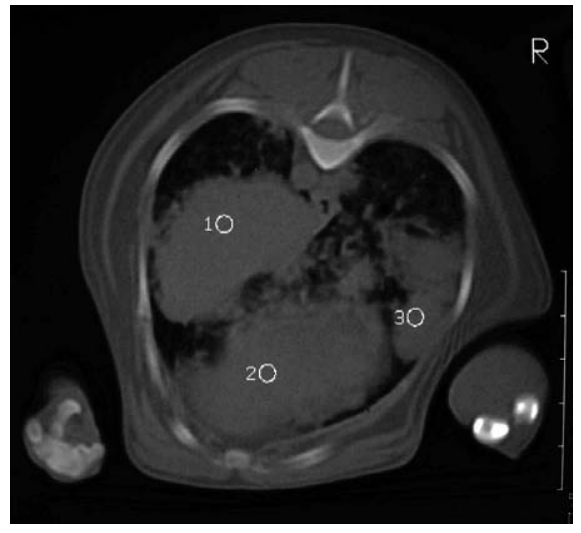

Fig. 2. CT scan of the thorax showing bilateral, wedge-shaped opacities.

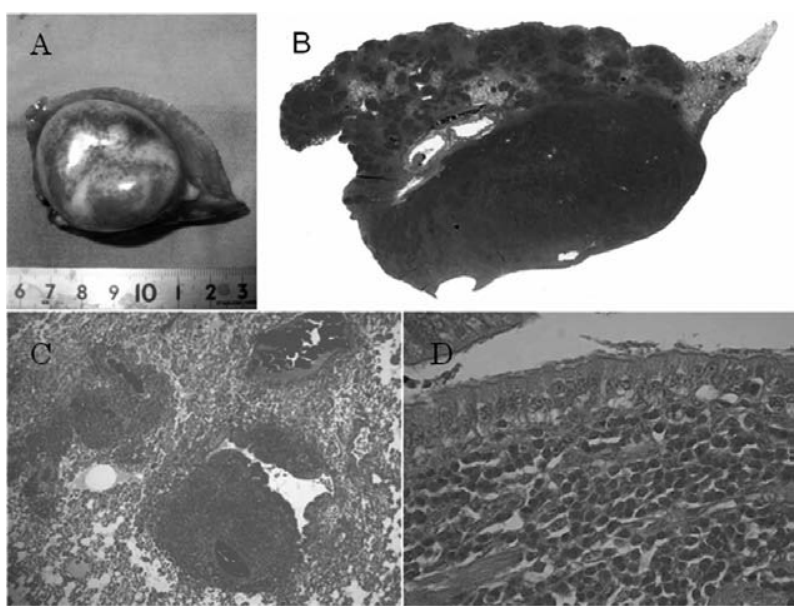

Fig. 3. Enucleated tumor and histopathological examination. (A) Macroscopic view of the lung nodule. (B) The mass consists of diffuse infiltration and proliferation of lymphoid cells. (C) Angiocentric pattern is noted surrounding lung parenchyma. (D) Higher magnification reveals infiltrate of lymphoid cells, including the large atypical mononuclear cells around the bronchiole.

sion. Enucleated firm, cream-colored nodular mass was found and that was $5 \mathrm{~cm}$ in diameter (Fig. 3A).

Histopathology revealed that an atypical angiocentric infiltration and proliferation of pleomorphic mononuclear cells that spread into the adjacent alveolar parenchyma (Fig. $3 \mathrm{~B}$ and $3 \mathrm{C}$ ). The infiltrating mononuclear cells resembled large lymphoid cells (Fig. 3D). Based on the histopathological findings, a diagnosis of pulmonary LYG was made.

The dog was started on a multiple drug protocol based on modified UW-25. This protocol consisted of vincristine $\left(0.5 \mathrm{mg} / \mathrm{m}^{2} \mathrm{iv}\right)$, cyclophosphamide $\left(250 \mathrm{mg} / \mathrm{m}^{2} \mathrm{iv}\right)$, doxorubicin $\left(30 \mathrm{mg} / \mathrm{m}^{2} \mathrm{iv}\right)$, and prednisone, without L-asparaginase. Seven days after the first administration of vincristine, the pulmonary mass and sterna lymphadenopathy markedly decreased in size and almost disappeared (Fig. 4). After treatment with chemotherapy protocol for 25 weeks, the

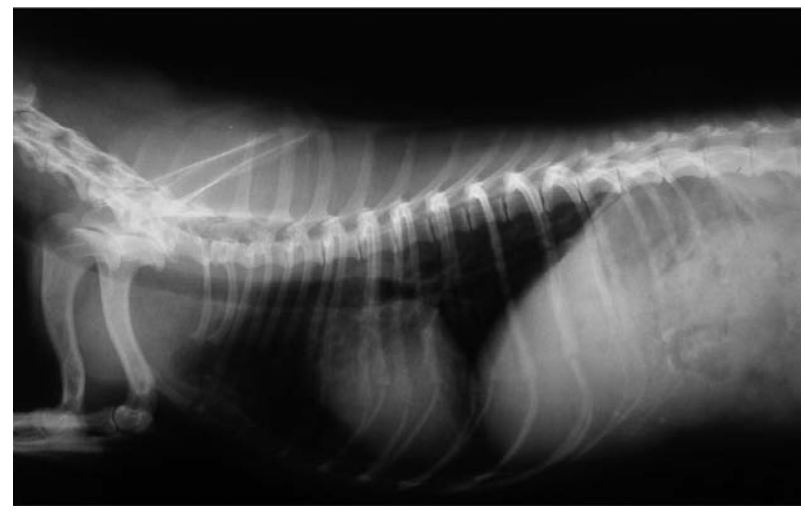

Fig. 4. Thoracic radiograph at 7 days after the first administration of vincristine (left lateral).

condition of dog was very well and the dog was considered in complete remission. The dog was scheduled to be monitored at routine appointments including clinical examination, $\mathrm{CBC}$, serum chemistry panel, thoracic radiographs and abdominal ultrasound.

On day 786 after presentation, a resurgence of clinical signs, pyrexia, dyspnea, diarrhea, and vomit, occurred. Ultrasound of the abdomen revealed abdominal lymphadenopathy and gastric wall thickening. The dog was tried to treat on a modified UW-25 again. However, because these treatments were not effective on this dog, administration of mitoxantrone $\left(5 \mathrm{mg} / \mathrm{m}^{2}\right)$ was started from day 830 once a month and clinical improvement and partial remission of abdominal lymphadenopathy was achieved. From day 1,014 , dyspnea, diarrhea and severe vomit were observed. The dog finally died 1,022 days after the first presentation. Complete necropsy was conducted.

Necropsy revealed lobar pneumonia with angiocentric infiltration of lymphocytic cells in the pulmonary parenchyma. Clustered lymphocytes were also observed in lamina propria and submucosa of stomach. Immunohistochemical examination of the lung revealed many CD79a positive B cells and a few CD3 positive T cells in the lymphoid cells around the blood vessels (Fig. 5A and 5B). Those angiocentric cells were negative for macrophage/histiocyte markers, AM-3K and lysozyme (Fig. 5C and 5D). Polymerase chain reaction (PCR) assays for the immunoglobulin and T-cell receptor genes were performed according to the protocol described by Burnett et al. [3] and Valli et al. [21]. The clonality for B lymphocyte was detected from lung tissues.

The typical character described in human and canine pulmonary LYG include multiple, bilaterally nodular masses $[10,17]$. Moreover, LYG is an angiocentric and angiodestructive lymphoproliferative disease involving extranodal sites $[2,10]$. In most patients with human LYG, similar cellular infiltrates are found in other organs such as skin, kidney, and central nervous system [10]. Canine LYG also often metastasizes to the regional lymph nodes, liver, and spleen [17]. Moreover, the case of human LYG affecting 


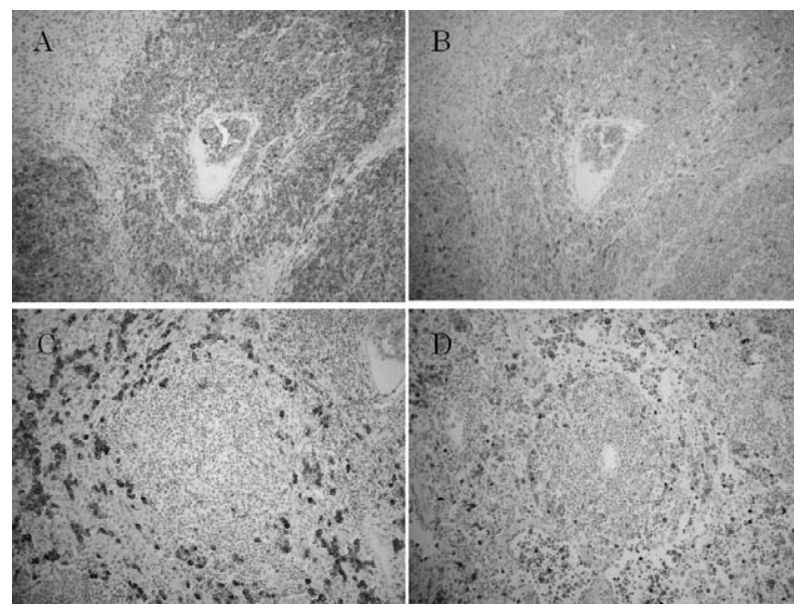

Fig. 5. Immunohistochemistry of the LYG. Angiocentric infiltrating mononuclear cells were mostly CD79a positive B cells (A) and a few $\mathrm{CD} 3$ positive $\mathrm{T}$ cells (B), but they lacked expressions of AM-3K (C) and lysozyme (D).

the gastrointestinal tract lesions was described, and demonstrated there was the typical angiocentric and angioinvasive legions of LYG in the gastric lesion [18]. In our case, however, although infiltration of lymphocytes was observed in lamina propria and submucosa of stomach, the typical angiocentric and angioinvasive legions were not observed. It is unclear whether the present case could be a LYG involving the gastrointestinal tract.

On cytological and histopathological analysis, the present case needs to be differentiated from histiocytic tumors. Immunohistochemically, the angiocentric infiltrating mononuclear cells were mostly CD79 positive B cells and a few CD3 positive T cells. Moreover, they lacked expressions of AM-3K and lysozyme. Therefore, the immunophenotype supports this case was not histiocytic tumor.

Pulmonary lymphoma may be difficult to distinguish from LYG, since human LYG may develop aggressive diffuse large B-cell lymphoma [10]. However, it was reported that diffuse multinodular patterns of pulmonary lymphoma are rare in canine [1] and human [4]. Moreover, although one report of canine pulmonary lymphoma revealed that cytological findings were consistent with lymphoma [15], this case revealed the presence of large lymphohistiocytic cells and small lymphocytes with occasional neutrophils and plasma cells, that were consistent with LYG previously reported $[2,12]$. Finally, histopathology in the present case was in agreement with histologic criteria for LYG, namely a nodular infiltrate of an angiocentric/angiodestructive lymphoid infiltrate within the pulmonary parenchyma [2, 12]. Those results supported that this case was diagnosed as a LYG.

Human LYG comprised of a usually small number of B cells admixed with prominent $\mathrm{T}$ cell infiltrates, and moreover, this is considered a form of T-cell rich B cell lymphoma on the basis of histopathological features and immunophenotypic analysis [8]. The phenotype of canine LYG is now controversial because immunohistochemical analyses of the canine LYG have been documented in only a few papers $[14,16,20]$. In one report, canine LYG has been associated with malignant $\mathrm{T}$-cell type lymphocytes and is considered a T-cell lymphoma [20]. Moreover, the presence of clonally expanded T-cell was revealed by polymerase chain reaction (PCR) in a CD3-positive canine cutaneous LYG, recently [19]. The other report of a pulmonary case, however, showed that there were both CD3 positive $\mathrm{T}$ cells and CD79 positive B cells [16]. The immunophenotypic analysis showed that canine cutaneous LYG had both neoplastic T- and B-cells [14]. Same as later reports, the present pulmonary LYG had both $\mathrm{T}$ cell and $\mathrm{B}$ cell lineage. Moreover, B cell monoclonality was revealed by PCR in this case. Therefore, the present dog had features similar to those of canine LYG and human LYG.

Canine LYG is very difficult to diagnose, and treatment has not been attempted actively [17]. In human and veterinary medicine, the LYG was diagnosed by open biopsy and histological evaluation of tissues [6, 10]. Same as those reports, this case could not be diagnosed by cytological examination, due to the marked cytologic variation of sample collection, and thus open lung biopsy was needed. In human LYG, it has been shown that a good response and long-term survival may possibly be obtained through appropriate chemotherapy [9], and the present canine case also could survive for long-term by CHOP chemotherapy. Therefore, the open lung biopsy is necessary to diagnose canine LYG and to treat properly.

Because canine LYG is rare, only a few treatment studies including prednisone, COP and COAP have been conducted and there is no standard treatment $[2,17]$. In human medicine, however, the patient with LYG was treated by a more aggressive combination chemotherapy regimen utilizing doxorubicin $[5,9]$. To the best of our knowledge, this was the first case of canine pulmonary LYG treated by CHOP chemotherapy, and could have long-term survival. Therefore, CHOP therapy may be effective for canine LYG, as applied for human LYG.

Rescue protocols have not been reported in canine and human LYG. After CHOP was not effective on this dog, administration of mitoxantrone was started once a month and clinical improvement and partial remission was achieved. This drug was used as rescue protocols of canine lymphoma [13]. The results of this report demonstrate that mitoxantrone may be an effective rescue protocol for dogs with LYG.

Further studies including immunohistochemical analyses, PCR clonality analysis and clinical trials will be necessary to confirm the benefits of CHOP and mitoxantrone in the management of canine LYG.

ACKNOWLEDGMENT. We thank Dr. Y. Kagawa of NORTH LAB, Sapporo, for her technical supports and valuable discussion. 


\section{REFERENCES}

1. Ackerman, N. and Madewell, B. R. 1980. Thoracic and abdominal radiographic abnormalities in the multicentric form of lymphosarcoma in dogs. J. Am. Vet. Med. Assoc. 176: 36-40.

2. Berry, C. R., Moore, P. F., Thomas, W. P., Sisson, D. and Koblik, P. D. 1990. Pulmonary lymphomatoid granulomatosis in seven dogs (1976-1987). J. Vet. Intern. Med. 4: 157-166.

3. Burnett, R. C., Vernau, W., Modiano, J. F., Olver, C. S., Moore, P. F. and Avery, A. C. 2003. Diagnosis of canine lymphoid neoplasia using clonal rearrangements of antigen receptor genes. Vet. Pathol. 40: 32-41.

4. Colby, T. V. and Carrington, C. B. 1982. Pulmonary lymphomas simulating lymphomatoid granulomatosis. Am. J. Surg. Pathol. 6: 19-32.

5. Drasga, R. E., Williams, S. D., Wills, E. R., Roth, L. M. and Einhorn, L. H. 1984. Lymphomatoid granulomatosis. Successful treatment with CHOP combination chemotherapy. Am. J. Clin. Oncol. 7: 75-80.

6. Fitzgerald, S. D., Wolf, D. C. and Carlton, W. W. 1991. Eight cases of canine lymphomatoid granulomatosis. Vet. Pathol. 28: 241-245.

7. Garrett, L. D., Thamm, D. H., Chun, R., Dudley, R. and Vail, D. M. 2002. Evaluation of a 6-month chemotherapy protocol with no maintenance therapy for dogs with lymphoma. J. Vet. Intern. Med. 16: 704-709.

8. Guinee, D. G. Jr., Perkins, S. L., Travis, W. D., Holden, J. A., Tripp, S. R. and Koss, M. N. 1998. Proliferation and cellular phenotype in lymphomatoid granulomatosis: implications of a higher proliferation index in B cells. Am. J. Surg. Pathol. 22: 1093-1100.

9. Jaffe, E. S. and Wilson, W. H. 1997. Lymphomatoid granulomatosis: pathogenesis, pathology and clinical implications. Cancer Surv. 30: 233-248.

10. Katzenstein, A. L., Carrington, C. B. and Liebow, A. A. 1979. Lymphomatoid granulomatosis: a clinicopathologic study of 152 cases. Cancer 43: 360-373.

11. Liebow, A. A. 1972. Lymphomatoid Granulomatosis. Calif.
Med. 116: 48-49.

12. Liebow, A. A., Carrington, C. R. and Friedman, P. J. 1972. Lymphomatoid granulomatosis. Hum. Pathol. 3: 457-558.

13. Lucroy, M. D., Phillips, B. S., Kraegel, S. A., Simonson, E. R. and Madewell, B. R. 1998. Evaluation of single-agent mitoxantrone as chemotherapy for relapsing canine lymphoma. $J$. Vet. Intern. Med. 12: 325-329.

14. Magi, G. E., Di Pasquale, S. and Renzoni, G. 2009. Cutaneous lymphomatoid granulomatosis (angiotropic lymphoma) in a dog: immunophenotyping analysis. Vet. Res. Commun. 33: 145-147.

15. McKay, L. W., Levy, J. K. and Thompson, M. S. 2004. What is your diagnosis? Pulmonary lymphoma. J. Am. Vet. Med. Assoc. 224: $1587-1588$.

16. Park, H. M., Hwang, D. N., Kang, B. T., Jung, D. I., Song, G. S., Lee, S. J., Yhee, J. Y., Yu, C. H., Doster, A. R. and Sur, J. H. 2007. Pulmonary lymphomatoid granulomatosis in a dog: evidence of immunophenotypic diversity and relationship to human pulmonary lymphomatoid granulomatosis and pulmonary Hodgkin's disease. Vet. Pathol. 44: 921-923.

17. Postorino, N. C., Wheeler, S. L., Park, R. D., Powers, B. E. and Withrow, S. J. 1989. A syndrome resembling lymphomatoid granulomatosis in the dog. J. Vet. Intern. Med. 3: 15-19.

18. Rubin, L. A., Little, A. H., Kolin, A. and Keystone, E. C. 1983. Lymphomatoid granulomatosis involving the gastrointestinal tract. Two case reports and a review of the literature. Gastroenterology 84: 829-833.

19. Shimazaki, T., Nagata, M., Goto-Koshino, Y., Tsujimoto, H. and Shirota, K. 2010. A case of canine lymphomatoid granulomatosis with cutaneous lesions. J. Vet. Med. Sci. 72: 10671069.

20. Smith, K. C., Day, M. J., Shaw, S. C., Littlewood, J. D. and Jeffery, N. D. 1996. Canine lymphomatoid granulomatosis: an immunophenotypic analysis of three cases. J. Comp. Pathol. 115: $129-138$.

21. Valli, V. E., Vernau, W., de Lorimier, L. P., Graham, P. S. and Moore, P. F. 2006. Canine indolent nodular lymphoma. Vet. Pathol. 43: 241-256. 